\title{
Action du Dichlorophène sur les Cestodes et les Nématodes du Poulet
}

\author{
por J. GUILHON et M. GRABER
}

Dans un travail antérieur nous avons étudié l'action du Dichlorophène ou dihydroxydichlorodiphényl-méthane sur les Cestodes parasites du Mouton. Dans celui-ci nous exposons les résultats des recherches entreprises pour déterminer la valeur anthelminthique du même corps à l'égard des principaux Cestodes ef Nématodes rencontrés dans l'intestin grêle du Poulet en milieu tropical (Tchad).

\section{I. - MATÉRIEL}

Les expériences furent effectuées sur 216 poulets, agés de quelques mois, nés et élevés dans la région de Fort-Lamy, renfermant dans leur tube digestif six espèces de Cestodes et 3 espèces de Nématodes. Dans 40 p. 100 des cas, les neuf espèces se trouvaient associées très différemment et en proportion variable (tableau 1).

- Les poulets diversement parasités offraient un matériel très varié sur lequel la polyvalence anthelminthique peut être étudiée plus fructueusement.

\section{II. - MÉTHODE}

Les traitements furent entrepris à plusieurs époques sur 4 années :

- octobre-novembre 1959,

- mai 1960,

- février-avril 1961,

- octobre-novembre 1961,

- mars-av́ril-mai 1962.

Rev. Elev. Méd. vét. pays trop., 1963; 16, no 3.

Reçu pour publication, juillet 1963.
Par cet étalement dans le temps, il a été possible d'apprécier l'efficacité de l'anthelminthique à diverses périodes, c'est-à-dire en saison favorable (septembre à février) durant laquelle la nourriture est abondante et les animaux en bon état et en saison défavorable (de février à août).

Les sujets choisis se reposèrent pendant environ 36 à 48 heures et furent placés dans des cagesgrillagées sur des supports à $25 \mathrm{~cm}$ du sol de façon à recueillir les parasites éliminés avec les fèces durant, l'expérience.

Aux poulets soumis à une diète de 20 à 24 heures le Dichlorophène est administré par vole orale dans des capsules de gélatine*, aux doses uniques de $30 \mathrm{mg}$ à $1 \mathrm{~g} / \mathrm{kg}$, ou répétées deux ou trois fois à 24 heures d'intervalle.

Après le traitement les fèces sont recueillies, broyées dans l'eau ef minutieusement examinées pour en extraire les helminthes évacués.

Les sujets sont sacrifiés 5 à 10 jours après l'intervention pour effectuer la récolte des vers restants. Les premières parties de l'intestin grêle sont, en outre, raclées sur une longueur d'environ $25 \mathrm{~cm}$ ef le prélèvement est examiné minutieusement entre lame et lamelle. Cette façon de faire est absolument indispensable pour retrouver les immatures, les scolex de Choonotaenia infundibulum et des diverses espèces de Railliefina qui demeurent souvent fixés à lá muqueuse intestinale, bien que leurs strobiles aient cédé à l'action anthelminthique. Enfin, les. Cestodes récoltés dans les excréments après traitement ef ceux qui sont trouvés après sacrification sont pesés séparément. La différence

* A quatre coqs il fut présenté mélangé à du mil légèremen humecté : deux l'ont absorbé, les deux autres l'ont refusé en partie. 
TABLEAU 1.

\begin{tabular}{|c|c|c|c|}
\hline Types d'association & Espèces trouvées & $\begin{array}{l}\text { Nombre } \\
\text { de sujets }\end{array}$ & Proportions \\
\hline $\begin{array}{l}2 \text { espèces } \\
\text { A) Cesiodes }\end{array}$ & $\begin{cases}R . \text { tetragona } & + \text { R. cesticillus } \\
R . t & +R . \text { echinobothrida } \\
R . t & +C h . \text { infundibulum } \\
R . t & +H . \text { carioca } \\
\text { R. echinob. } & + \text { Ch. infundibulum } \\
\text { R. echinob. }+ \text { H. carioca }\end{cases}$ & $\begin{array}{r}6 \\
4 \\
1 \\
12 \\
1 \\
3\end{array}$ & $65: 73,8$ p. 100 \\
\hline $\begin{array}{l}3 \text { espèces } \\
\text { A) } 3 \text { Cestodes }\end{array}$ & $\begin{array}{l}\text { R. }+ \text { + Ascaridia styph. } \\
\text { R. }+ \text { + Subulura brumpti } \\
\text { R. echinob. + Subulura brumpti } \\
\text { R. echinob. + Ascaridia styph. } \\
\text { R. cesticilius + Subulura brumpti } \\
\text { R. cesticilius + Ascoridia styph. } \\
\text { H. carioca + Ascaridia styphlocerca } \\
\text { H. corioca + Subulura brumpti } \\
\text { R. }++ \text { R. cest. + H. carioca } \\
\text { R. }+ \text { R. echin. + H. corioca } \\
\text { R. }+ \text { Ch. infund. + H. carioca }\end{array}$ & $\begin{array}{r}3 \\
14 \\
2 \\
1 \\
2 \\
2 \\
3 \\
3 \\
1 \\
1 \\
1\end{array}$ & \\
\hline $\begin{array}{l}\text { B) } 2 \text { Cestodes } \\
1 \text { Nématode }\end{array}$ & 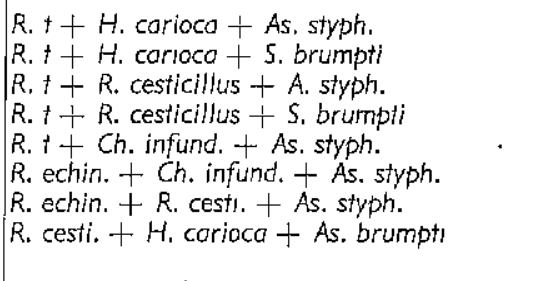 & $\begin{array}{l}3 \\
1 \\
2 \\
1 \\
2 \\
1 \\
1 \\
1\end{array}$ & $16: 18,1$ p. 100 \\
\hline $\begin{array}{l}2 \text { Nématodes } \\
4 \text { espèces } \\
\text { A) Cestodes } \\
1 \text { Nématode } \\
\text { B) } 2 \text { Cestodes } \\
2 \text { Nématodes }\end{array}$ & $\left\{\begin{array}{l}\text { H. carioca + As. styph. }+ \text { S. brumpti } \\
\text { R. }++ \text { R. cest. + H. car. }+ \text { S. brumpti } \\
\text { R. }+ \text { + R. cest. + R. echin. }+ \text { S. brumpti } \\
\text { R. }+ \text { H. car. + C. digono. + S. brump. } \\
\text { R. }+ \text { R. cest. + Acuaria sp. + S. brum. } \\
\text { R. ech. + H. car. + As. styph. + S. brumpti }\end{array}\right.$ & $\begin{array}{l}1 \\
2 \\
1 \\
1 \\
1 \\
1\end{array}$ & $7: 8,1$ p. 100 \\
\hline
\end{tabular}

pondérale permet d'apprécier l'efficacité du médicament utilisé, compte tenu des renseignements fournis par le raclage de la muqueuse.

Dans ces conditions expérimentales les résultats obtenus avec le Dichlorophène administré sous deux formes (pur et à 98 p. 100 de pureté) sont groupés dons plusieurs tableaux ci-inclus (II-III-IV-V-VI-VII-VIII-IX). II ressort de leur lecture qu'il agit très différemment sur les Nématodes ef les Cestodes. Son action est nulle à l'égard des premiers et nuancés vis-à-vis des seconds suivant les espèces en cause è les doses employées. Certaines espèces comme Hymenolepis carioca sont réfractaires quelle que soit la dose utilisée et répétée 2 ou 3 fois à 24 heures d'intervalle.

Les autres espèces sont diversement sensibles:
Raillietıno cesticillus est évacué soit avec une dose unique de $800 \mathrm{mg} / \mathrm{kg}$, soit avec celle de $500 \mathrm{mg} / \mathrm{kg}$ répétée trois fois et $R$. tetragona ainsi que $R$. echinobothrido avec celle de $300 \mathrm{mg} / \mathrm{kg}$. Les formes immatures des trois Cestodes résistent à toufes les doses utilisées aussi bien uniques que répétées.

Si on compare les résultats que nous avons constatés avec ceux des auteurs étrangers (Américains, Allemands, Japonais) qui ont étudié l'action du Dichlorophène sur les Cestodes parasites du Poulet, quelques divergences apparaissent comme il est facile de le remarquer $d$ après les indications groupées dans le tableau $X$.

Elles peuvent provenir du mode d'administration, de la plus ou moins grande pureté du médicament utilisé ou de la plus ou moins 
TABLEAU N* II

Corpa \& 98 p.100 - Paraites adultes - Ioess uniquas - Diète de 20 heurges.

\begin{tabular}{|c|c|c|c|c|c|c|}
\hline $\begin{array}{c}\text { Dosis } \\
(\mathrm{me} / \mathrm{kg})\end{array}$ & $\begin{array}{c}\text { Nombra } \\
d^{+} \text {eanztiaur }\end{array}$ & $\begin{array}{c}\text { Poida des animaux } \\
\left(\text { (on } \varepsilon^{\prime}\right)\end{array}$ & Parasites en canse. & $\begin{array}{l}\text { Nombre d'animaux to- } \\
\text { talement déparabités }\end{array}$ & $\begin{array}{c}\text { Efficacisté } \\
(\mathrm{p} .100)\end{array}$ & Scolex. \\
\hline $\begin{array}{l}30 \\
50 \\
60 \\
80\end{array}$ & $\begin{array}{l}1 \\
1 \\
1 \\
1\end{array}$ & $\begin{array}{r}1.570 \\
980 \\
802 \\
635\end{array}$ & $\begin{array}{l}\text { Patliletina tetragona } \\
\text { dacaridia styphlocerca } \\
\text { Ralliletina tetragona } \\
\text { Raillietina tetragona }\end{array}$ & $\begin{array}{l}0 \\
0 \\
0 \\
0\end{array}$ & $\begin{array}{r}50 \\
0 \\
16 \\
0\end{array}$ & $\begin{array}{l}+4 \\
++++ \\
+++\end{array}$ \\
\hline 120 & $\begin{array}{l}4 \\
2 \\
2\end{array}$ & $\begin{array}{l}612-835-930-800 \\
930-800 \\
930-800\end{array}$ & $\begin{array}{l}\text { Reililetina tetragons } \\
\text { hymenolepla carioca } \\
\text { Asceridia styphlocerea }\end{array}$ & $\begin{array}{l}4 \\
0 \\
0\end{array}$ & $\begin{array}{r}100 \\
0 \\
0\end{array}$ & $\begin{array}{l}0 \\
+1+4\end{array}$ \\
\hline 160 & 1 & 630 & Subulusa brumpti & 0 & $\overline{0}$ & \\
\hline 175 & $\begin{array}{l}3 \\
3 \\
1 \\
1 \\
1 \\
1\end{array}$ & $\begin{array}{l}680-520-620 \\
540-600-740 \\
670 \\
620 \\
560 \\
950\end{array}$ & 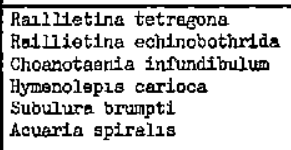 & $\begin{array}{c}3 \text { our } 4 \\
0 \\
0 \\
0 \\
0 \\
0\end{array}$ & $\begin{array}{r}94 \\
25 \\
0 \\
0 \\
0 \\
0\end{array}$ & $\begin{array}{l}+ \\
+++ \\
+++ \\
+++\end{array}$ \\
\hline 200 & $\begin{array}{l}3 \\
1 \\
1 \\
2 \\
1 \\
1 \\
1\end{array}$ & $\begin{array}{l}880-700-680 \\
720 \\
700 \\
720-880 \\
720 \\
720 \\
680\end{array}$ & 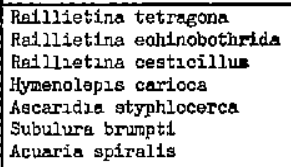 & $\begin{array}{c}2 \text { aux } 3 \\
0 \\
0 \\
0 \\
0 \\
0 \\
0\end{array}$ & $\begin{array}{r}93 \\
0 \\
0 \\
0 \\
0 \\
0 \\
0\end{array}$ & $\begin{array}{l}+ \\
+++ \\
+++ \\
++1\end{array}$ \\
\hline 225 & $\begin{array}{l}3 \\
4 \\
2 \\
1\end{array}$ & $\begin{array}{l}660-780-660 \\
780-800-660-660 \\
800-780 \\
780\end{array}$ & $\begin{array}{l}\text { Rail]zetina tatragona } \\
\text { Hymenolepis carioca } \\
\text { Ascarzal styphlocerca } \\
\text { Subulura brumpti }\end{array}$ & $\begin{array}{c}1 \text { gle } 3 \\
0 \\
0 \\
0\end{array}$ & $\begin{array}{r}87 \\
0 \\
0 \\
0\end{array}$ & +4 \\
\hline 250 & $\begin{array}{l}4 \\
2 \\
1 \\
3\end{array}$ & $\begin{array}{l}640-880-860-720 \\
780-860 \\
380 \\
380-880-620\end{array}$ & $\begin{array}{l}\text { Rezllietina tetragona } \\
\text { Hyquenolepis camoca } \\
\text { Ascarzdia styphlocerca } \\
\text { Subulure brumgti }\end{array}$ & $\begin{array}{c}1 \text { sur } 4 \\
0 \\
0 \\
0\end{array}$ & $\begin{array}{r}30 \\
0 \\
0 \\
0\end{array}$ & $\begin{array}{l}++ \\
+1+\end{array}$ \\
\hline 300 & $\begin{array}{l}6 \\
1 \\
4 \\
3 \\
1\end{array}$ & $\begin{array}{l}595-690-607-530 \\
600-570- \\
765- \\
600-1020-700-690 \\
680-550-690 \\
660\end{array}$ & 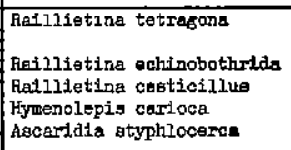 & $\begin{array}{c}6 \text { oux } 6 \\
1 \text { oux } 9 \\
2 \text { our } 4 \\
0 \\
0\end{array}$ & $\begin{array}{r}100 \\
100 \\
76 \\
0 \\
0\end{array}$ & $\begin{array}{l}0 \\
0 \\
+ \\
++1+\end{array}$ \\
\hline 320 & $\begin{array}{l}1 \\
1\end{array}$ & $\begin{array}{l}630 \\
630\end{array}$ & $\begin{array}{l}\text { Hywenolepia carioca } \\
\text { Lscarlaia etyphlocerca }\end{array}$ & $\begin{array}{l}0 \\
0\end{array}$ & $\begin{array}{l}0 \\
0\end{array}$ & $+1+$ \\
\hline 400 & $\begin{array}{l}7 \\
5 \\
3 \\
6 \\
2 \\
8 \\
2\end{array}$ & $\begin{array}{l}510-620-600-600-700- \\
700-420 \\
730-700-600-700-680 \\
700-680-420 \\
730-770-520-700-700-700 \\
620-520 \\
750-700-600-700- \\
700-680-420-600 \\
700-420\end{array}$ & 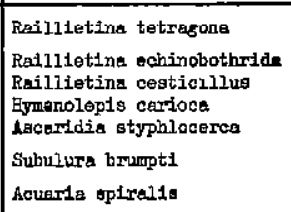 & $\begin{array}{c}7 \text { our } 7 \\
5 \text { our } 5 \\
0 \\
0 \\
0 \\
0 \\
0\end{array}$ & $\begin{array}{r}100 \\
100 \\
0 \\
0 \\
0 \\
0 \\
0\end{array}$ & $\begin{array}{l}0 \\
0 \\
+++ \\
+++\end{array}$ \\
\hline 500 & $\begin{array}{l}5 \\
1 \\
3 \\
1\end{array}$ & $\begin{array}{l}665-690-760-760-600 \\
610 \\
660-600-780 \\
560\end{array}$ & 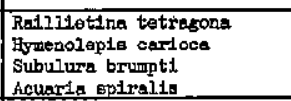 & $\begin{array}{c}5 \text { our } 5 \\
0 \\
0 \\
0 \\
\end{array}$ & $\begin{array}{r}100 \\
0 \\
0 \\
0 \\
\end{array}$ & 0 \\
\hline 600 & 1 & 430 & Finililietuna tetragana & 1 our 1 & 100 & 0 \\
\hline 800 & 1 & $\begin{array}{l}600 \\
600 \\
\end{array}$ & $\begin{array}{l}\text { Hedllietina cesticiliue } \\
\text { Subulura brumpti }\end{array}$ & $\begin{array}{c}\text { sur } 1 \\
0\end{array}$ & $\begin{array}{r}100 \\
0 \\
\end{array}$ & 0 \\
\hline 1.000 & $\frac{1}{1}$ & 400 & Fatliletins totregona & 1 our 1 & 100 & 0 \\
\hline
\end{tabular}


TABIERAU NO III

Corps la 98 p.100 - Parasites adultes - Dasas répétées doux folo à 24 heures d'intervalle

\begin{tabular}{|c|c|c|c|c|c|c|}
\hline $\begin{array}{c}\text { Doges } \\
(\mathrm{mg} / \mathrm{kg})\end{array}$ & $\begin{array}{l}\text { Nomibre } \\
\text { d'antinaux }\end{array}$ & $\begin{array}{c}\text { Poide des animaux } \\
(\text { en g.) }\end{array}$ & - Parasitas en cellse & $\begin{array}{l}\text { Nombre d'anzuaux, to- } \\
\text { talement déparasités }\end{array}$ & $\begin{array}{l}\text { Efficacité } \\
(\mathrm{p} .100)\end{array}$ & Scoler \\
\hline 400 & 2 & $760-660$ & Rallitetina tetragona & 2 sur 2 & 100 & D \\
\hline 400 & $\bar{i}$ & 660 & Acuaria aplrall,s & $\overline{0}$ & $\overline{0}$ & \\
\hline 500 & $\begin{array}{l}3 \\
1 \\
1\end{array}$ & $\begin{array}{l}610-800-710 \\
630 \\
730\end{array}$ & $\begin{array}{l}\text { Failliotina tetragona } \\
\text { haillietina echlnobothrida } \\
\text { hevarta splralis }\end{array}$ & $\begin{array}{c}3 \text { sur } \\
1 \text { sur } \\
0\end{array}$ & $\begin{array}{c}100 \\
100 \\
0\end{array}$ & $\begin{array}{l}0 \\
0\end{array}$ \\
\hline$\overline{800}$ & 1 & 570 & Subulura brumpti & 0 & $\overline{0}$ & \\
\hline 1.000 & $T$ & 655 & Subuilura brumpti & 0 & $\overline{0}$ & \\
\hline
\end{tabular}

TABLEAU NO IV

Corps à 98 p.100 - Paragites adultea - Doses répétées trois fois à 24 heures d'intervelle

\begin{tabular}{|c|c|c|c|c|c|c|}
\hline 300 & $\begin{array}{l}3 \\
2 \\
1\end{array}$ & $\begin{array}{l}770-790-540 \\
600-570 \\
765\end{array}$ & $\begin{array}{l}\text { Rmilletina tetragona } \\
\text { Subulura brumpti } \\
\text { Acuaria spiralia }\end{array}$ & $\begin{array}{c}3 \sin 3 \\
0 \\
0\end{array}$ & $\begin{array}{c}100 \\
0 \\
0\end{array}$ & D \\
\hline 400 & $\begin{array}{l}1 \\
2 \\
1 \\
1\end{array}$ & $\begin{array}{l}640 \\
535-655 \\
695 \\
450\end{array}$ & $\begin{array}{l}\text { Ratilietine tetragona } \\
\text { Raillietina ochinobothrida } \\
\text { Bymenolepis cartoca } \\
\text { Subulura brumpti }\end{array}$ & $\begin{array}{c}1 \text { sur } \\
2 \text { sis } 2 \\
0 \\
0\end{array}$ & $\begin{array}{c}100 \\
100 \\
0 \\
0\end{array}$ & $\begin{array}{l}0 \\
0 \\
++1+\end{array}$ \\
\hline 500 & $\begin{array}{l}3 \\
1\end{array}$ & $\begin{array}{l}580-600-650 \\
600\end{array}$ & $\begin{array}{l}\text { Raillieting tetragona } \\
\text { Rallifetina cestialllus }\end{array}$ & $\begin{array}{l}3 \text { sir } 3 \\
1 \sin 1\end{array}$ & $\begin{array}{l}100 \\
100\end{array}$ & $\begin{array}{l}0 \\
0\end{array}$ \\
\hline 800 & $\begin{array}{l}1 \\
1 \\
1\end{array}$ & $\begin{array}{l}530 \\
530 \\
610\end{array}$ & $\begin{array}{l}\text { Ralliletina tetragons } \\
\text { Rallfletina echinobothrida } \\
\text { Subulura brumpti }\end{array}$ & 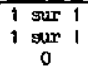 & $\begin{array}{c}100 \\
100 \\
0\end{array}$ & $\begin{array}{l}0 \\
0\end{array}$ \\
\hline 1.000 & 2 & $710-950$ & Raillietina echinobothrida & $2 \sin 2$ & 100 & $\overline{0}$ \\
\hline
\end{tabular}

\section{TABIBASU NO N}

Témalns.

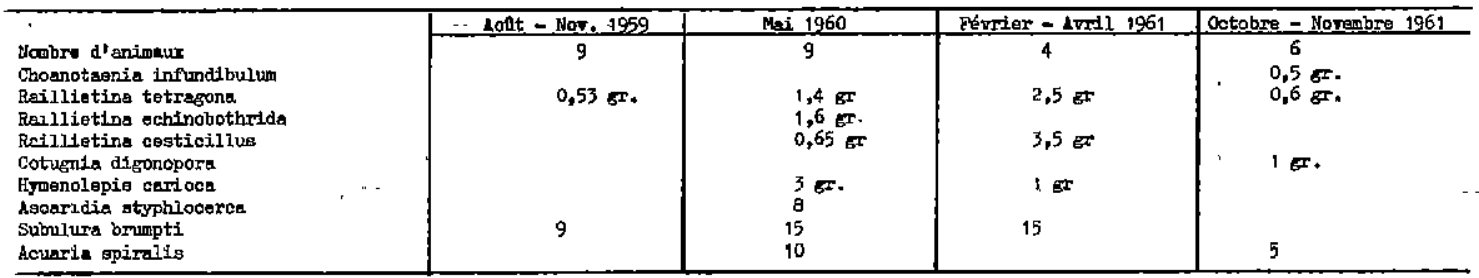


Retour au menu

TABLEAU SO VI

Corps ha 99 p.100 - Cestades immatures - Diète de 20 heures.

\begin{tabular}{|c|c|c|c|c|}
\hline $\begin{array}{c}\text { Doses } \\
(\mathrm{mg} / \mathrm{kg})\end{array}$ & $\begin{array}{l}\text { Hoglbre de poulets } \\
\text { traeltés }\end{array}$ & $\begin{array}{l}\text { Nombre do poulets } \\
\text { enoore parasités. }\end{array}$ & Parasites en cause (Nombre & $\overline{\text { ogyen })}$ \\
\hline 175 & 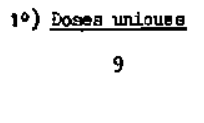 & $i_{i}^{1}$ & $\begin{array}{l}\text { Choanotanis infundibulum } \\
\text { Rallilietive tetragome } \\
\text { Radilietine echnobothrida }\end{array}$ & $\begin{array}{l}: 3 \\
: 1 \\
: 1\end{array}$ \\
\hline 200 & 7 & $\begin{array}{l}i \\
i \\
i\end{array}$ & $\begin{array}{l}\text { Raililiatina tetragona } \\
\text { Reillietina cestlcillua } \\
\text { Hymenolepis cerloca }\end{array}$ & $\begin{array}{l}1 \\
: 1 \\
: 10\end{array}$ \\
\hline 225 & 6 & 1 & $\begin{array}{l}\text { Fiaillietun cesticiplinus } \\
\text { Hyrmenolepis carloca }\end{array}$ & $\begin{array}{l}: 100 \\
: 30\end{array}$ \\
\hline 250 & 9 & $\begin{array}{l}1 \\
2 \\
3\end{array}$ & $\begin{array}{l}\text { Refilietina tetragona } \\
\text { Reillietina cesticillus } \\
\text { Hyonolepis carroca }\end{array}$ & $\begin{array}{l}1 \\
2 \\
5\end{array}$ \\
\hline 300 & 29 & $\begin{array}{l}4 \\
1 \\
2\end{array}$ & $\begin{array}{l}\text { Rallilietina tetrazona } \\
\text { Reililietine echiobothrida } \\
\text { Ralilietina cesticlilus }\end{array}$ & $\begin{array}{l}: 3 \\
: 1 \\
: 1\end{array}$ \\
\hline 400 & 29 & $\begin{array}{l}4 \\
2 \\
3\end{array}$ & $\begin{array}{l}\text { Raillietina tetragana } \\
\text { Ratllietina costic1llus } \\
\text { Hymenolepis carioce }\end{array}$ & $\begin{array}{l}: 2 \\
: 2 \\
: 2\end{array}$ \\
\hline 500 & 25 & $\begin{array}{l}2 \\
5 \\
1\end{array}$ & $\begin{array}{l}\text { Ralilietina tetragona } \\
\text { Roillietine cesticillus } \\
\text { Reillietune ochnobothride }\end{array}$ & $\begin{array}{l}76 \\
: 5 \\
: 1 \\
\end{array}$ \\
\hline 600 & 3 & $\begin{array}{l}1 \\
1\end{array}$ & $\begin{array}{l}\text { Raillietine tetrafona } \\
\text { Reillietune cestlcillus }\end{array}$ & $: 9$ \\
\hline 800 & $\overline{3}$ & $\overline{1}$ & haflifetina tetragone & $: 1$ \\
\hline 1000 & 6 & $\begin{array}{l}1 \\
1\end{array}$ & $\begin{array}{l}\text { Raillietina tetragonn } \\
\text { Raillietine cesticillus }\end{array}$ & $\begin{array}{l}1 \\
: 1\end{array}$ \\
\hline
\end{tabular}

Tŕmoins (formes imatures) Nonbre moven de parasites.

20) Doses répétées deux follo à 24. heursa d'intervelle.

\begin{tabular}{|l|l|l|l|l|}
\hline 400 & 6 & 2 & Raillietina tetragona & 1 \\
\hline 500 & 6 & 2 & Relliletina cesticlllus & $: 2$ \\
\hline 800 & 3 & 1 & Ralliletina totragana & 1 \\
\hline
\end{tabular}

30) Doses répétóes trais fors à 24 heures dointercralle.

\begin{tabular}{|c|c|c|c|c|}
\hline 300 & 13 & $\begin{array}{l}2 \\
1\end{array}$ & $\begin{array}{l}\text { Raillietine tetragona } \\
\text { Retllietine ceoticillus }\end{array}$ & $\begin{array}{l}13 \\
: 1\end{array}$ \\
\hline 400 & 12 & $\begin{array}{l}4 \\
1 \\
3\end{array}$ & $\begin{array}{l}\text { fiellilietine tatragono } \\
\text { helllietina echinobathrida } \\
\text { hesilietina cesticillus }\end{array}$ & $\begin{array}{l}: 1 \\
: 1 \\
: 1 \\
\end{array}$ \\
\hline 500 & 6 & 2 & flaillietine tetragona & $t$ \\
\hline 800 & 3 & 1 & Choenotienis Infunalbulum & $;$ \\
\hline
\end{tabular}

\section{Animaux}

R.tetrezona ( 4 poulets) :

5 R.cesticilus ( 4 pouzets ) : 12 Choanoteenta Infumdtbulum (1 poulat) 6 Raillietina echinobothridn(1 poulet) 1

\section{TABLEAU $\mathrm{H*}$ VII}

Corps pur - Doses uniques - Diète de 20 Heures

\begin{tabular}{|c|c|c|c|c|c|c|}
\hline $\begin{array}{l}\text { Doses } \\
(\mathrm{mg} / \mathrm{kt})\end{array}$ & $\begin{array}{l}\text { Noribre } \\
\text { d'an1maux }\end{array}$ & $\begin{array}{c}\text { Pords des animeux } \\
\text { (en E.) }\end{array}$ & Parasi tes en cause & $\begin{array}{l}\text { Hombre d'andmaur to } \\
\text { talement déparagités }\end{array}$ & $\begin{array}{l}\text { Efficuat té } \\
(\mathrm{p} .100)\end{array}$ & Scolex \\
\hline 150 & $\begin{array}{l}3 \\
2 \\
1\end{array}$ & $\begin{array}{l}700-800-840 \\
700-800 \\
740\end{array}$ & $\begin{array}{l}\text { Raillietina tetregona } \\
\text { Hymenolepls carloca } \\
\text { Acueria spiralis }\end{array}$ & $\begin{array}{c}2 \text { घur } 3 \\
0 \\
0\end{array}$ & $\begin{array}{c}95.3 \\
0 \\
0\end{array}$ & +4 \\
\hline 200 & $\begin{array}{l}8 \\
2 \\
2 \\
4 \\
5 \\
1 \\
4\end{array}$ & $\begin{array}{l}880-700-700-620-520 \\
800-700-540 \\
700-580 \\
800-980-700-520 \\
880-380-680-700-540 \\
840 \\
800-920-620-520-\end{array}$ & $\begin{array}{l}\text { Retlilatina tetragona } \\
\text { Fieillzetina echinobothrida } \\
\text { Hyrenolepia carloca } \\
\text { Subulure brumpti } \\
\text { Ascaridia styphiocerce } \\
\text { Acuarda spiralis }\end{array}$ & $\begin{array}{c}6 \text { oxt } \theta \\
2 \text { gur } 2 \\
0 \\
0 \\
0 \\
0\end{array}$ & $\begin{array}{c}94,4 \\
100 \\
0 \\
0 \\
0 \\
0\end{array}$ & $\begin{array}{r}+ \\
0 \\
+++\end{array}$ \\
\hline 250 & $\begin{array}{l}11 \\
3 \\
3 \\
1\end{array}$ & $\begin{array}{l}780-800-800-500-960- \\
700-700-620-600-660-640 \\
700-640-640 \\
960-800-780 \\
600\end{array}$ & $\begin{array}{l}\text { Reillietina tetragone } \\
\text { Hytnonolepía cerroca } \\
\text { Subulura brumpti } \\
\text { Acuaria gpiralis } \\
\end{array}$ & $\begin{array}{c}9 \text { sur } 11 \\
0 \\
0 \\
0 \\
\end{array}$ & $\begin{array}{r}79 \\
0 \\
0 \\
0 \\
\end{array}$ & $\begin{array}{r}+ \\
++1+\end{array}$ \\
\hline 500 & $\begin{array}{l}3 \\
1 \\
1 \\
1 \\
\end{array}$ & $\begin{array}{l}700-700-680 \\
680 \\
700 \\
700\end{array}$ & $\begin{array}{l}\text { Raillietina tetragona } \\
\text { Raillietina ochinobothride } \\
\text { Ascoridia otyphloceres } \\
\text { Subulura brumpti }\end{array}$ & $\begin{array}{c}2 \text { aur } 3 \\
1 \text { our } 1 \\
0 \\
0\end{array}$ & $\begin{array}{r}86 \\
100 \\
0 \\
0 \\
\end{array}$ & $\stackrel{+}{0}$ \\
\hline 400 & $\begin{array}{l}2 \\
1 \\
1 \\
2 \\
\end{array}$ & $\begin{array}{l}600-680 \\
620 \\
620 \\
580-680 \\
\end{array}$ & $\begin{array}{l}\text { Raillietina tetrasona } \\
\text { Hywenolepis cariocs } \\
\text { Aocarioie styphlocerca } \\
\text { Subuluws brupt1 }\end{array}$ & $\begin{array}{c}2 \operatorname{sir} 2 \\
0 \\
0 \\
0 \\
\end{array}$ & $\begin{array}{r}100 \\
0 \\
0 \\
0 \\
\end{array}$ & $\begin{array}{c}0 \\
+++\end{array}$ \\
\hline 500 & $\begin{array}{l}2 \\
1 \\
1 \\
1\end{array}$ & $\begin{array}{l}660-760 \\
560 \\
650 \\
520\end{array}$ & 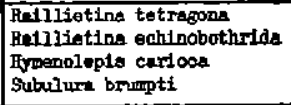 & $\begin{array}{c}2 \text { one } 2 \\
1 \text { our } 1 \\
0 \\
0\end{array}$ & $\begin{array}{c}100 \\
100 \\
0 \\
0\end{array}$ & $\begin{array}{r}0 \\
0 \\
+++\end{array}$ \\
\hline
\end{tabular}


TARLAR NO VII

Túnoino.

\begin{tabular}{|c|c|c|c|}
\hline & Dácembre 1961 & Mars 1962 & Arrll 1962 \\
\hline 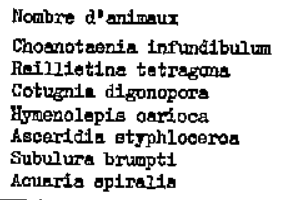 & $\begin{array}{l}4 \\
0,1 \mathrm{gr} . \\
0,6 \mathrm{gr} .\end{array}$ & $\begin{array}{cc}5 & \\
0,25 & \mathrm{ET} . \\
0,5 & \mathrm{Ex} . \\
0,5 & \mathrm{EF} . \\
12 & \end{array}$ & $\begin{array}{l}1 \mathrm{Gr} . \\
15\end{array}$ \\
\hline
\end{tabular}

TABIBAUU MO IX

Corps pur - Geatodos Inmatures - Diète de 20 herures

\begin{tabular}{|c|c|c|c|c|c|}
\hline 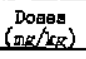 & 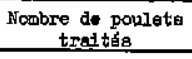 & $\begin{array}{l}\text { Nombre de poulets } \\
\text { encore parasités }\end{array}$ & Purasitas on cauge (Nom & a moyen) & $\begin{array}{l}\text { Ténouns (formes Inmatures) } \\
\text { Nombre moren de parasites }\end{array}$ \\
\hline 200 & 17 & $\begin{array}{r}4 \\
3 \\
2 \\
\end{array}$ & $\begin{array}{l}\text { Reilllietina totragone } \\
\text { Raillietina cesticillua } \\
\text { Hytuenolopis aarioos }\end{array}$ & $\begin{array}{rr}: & 6 \\
: 12 \\
: 10 \\
\end{array}$ & \multirow{5}{*}{ 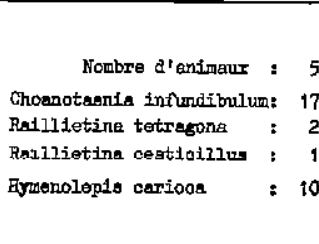 } \\
\hline 250 & 15 & $\begin{array}{r}2 \\
1 \\
\end{array}$ & $\begin{array}{l}\text { Refinietina tetragona } \\
\text { Choenotaonia infunfibulum }\end{array}$ & $\begin{array}{l}3 \\
1 \quad 2 \\
\end{array}$ & \\
\hline 300 & 4 & 1 & Rnllijetine cestrcillus & $\div 2$ & \\
\hline 400 & 9 & $\begin{array}{l}2 \\
1\end{array}$ & $\begin{array}{l}\text { Raillietine tetragona } \\
\text { Reillietina cesticillus }\end{array}$ & $\begin{array}{l}: 1 \\
: \quad 4\end{array}$ & \\
\hline 500 & 15 & 3 & Rafllietina tetragone & $: 1$ & \\
\hline
\end{tabular}

grande aftention accordée à la différence d'action sur les formes adultes et sur les formes immatures.

\section{III. - MODE D'ACTION}

Le Dichlorophène agit assez rapidement sur les Cestodes qui sont éliminés le plus souvent soit 24 heures ou au maximum 72 heures après son administration. Les parasites sont rejetés généralement amputés de leur scolex ou en menus fragments en voie de digestion.

Le Dichlorophène paraît annihiler le système de protection des Cestodes qui semblent alors subir l'attaque des sucs digestifs surtout au niveau de la partie antérieure de leur strobile. Malgré la rapide action du médicament plus cestocide que cestodifuge, les fragments de vers éliminés sont encore reconnaissables après éclaircissement ou coloration au carmin chlorhydrique.

\section{IV. - TOXICITÉ}

Aux doses thérapeutiques le Dichlorophène est bien supporté par les poulets. Leurs attitudes et leur comportement ne sont guère modifiés et l'appétit est conservé. Toutefois, si des doses élevées de l'ordre de $1 \mathrm{~g} / \mathrm{kg}$ jusqu'à $3,5 \mathrm{~g} / \mathrm{kg}$ sont administrées, elles provoquent une perte de poids durant quelques jours, puis la croissance ou l'engraissement se poursuit ultérieurement, normalement.

Les premiers accidents mortels se manifestent après l'ingestion de $4 \mathrm{~g} / \mathrm{kg}$, ce qui fait apparaître un coefficient chimiothérapique de l'ordre de 5 à 13, suivant qu'on utilise des doses de 300 à $800 \mathrm{mg} / \mathrm{kg}$. Les répercussions sur la ponte ne sont pas sensibles (SAWADA) et la chair des poulets traités a pu être consommée sans conséquences fâcheuses.

D'après les quelques travaux publiés à l'étranger sur l'action anthelminthique du Dichlorophène à l'égard des Helminthes du Poulet et les nôtres, il semble qu'on puisse admettre les indications suivantes :

$1^{\circ}$ Le Dichlorophène ou dihydroxydichlorodiphénylméthane manifeste une nette action anthelminthique à l'égard des Cestodes adultes tels que Roillietina tetragona et $R$. echinobothrida à la dose unique de $300 \mathrm{mg} / \mathrm{kg}$;

$2^{\circ}$ Des doses plus élevées $(700-800 \mathrm{mg} / \mathrm{kg}$ ) sont nécessaires pour intervenir sur Choanotaenia infundibulum et $R$. cesticillus ou inférieures (500 mg) mais répétées plusieurs fois:

30 Davainea proglottina et Hymenolepis carioca sont résistants même à des doses élevées : $800 \mathrm{mg}$ à $1 \mathrm{~g} / \mathrm{kg}$ et répétées ; 
TABLEAU $\times$

\begin{tabular}{|c|c|c|c|c|}
\hline Auteurs & Paras & Pays & $\begin{array}{l}\text { Doses } \\
(\mathrm{mg} / \mathrm{kg})\end{array}$ & Efficacité \\
\hline $\begin{array}{c}\text { KERR et GREEN, } 1953 \\
- \\
-\end{array}$ & $\begin{array}{c}\text { Roillietino cesticillus } \\
- \\
-\end{array}$ & $\begin{array}{l}\text { U.S. A. } \\
- \\
-\end{array}$ & $\begin{array}{r}50 \\
100 \\
150 \\
200\end{array}$ & $\begin{array}{r}5 \text { p. } 100 \\
22 \text { p. } 100 \\
27 \text { p. } 100 \\
3 \text { p. } 100\end{array}$ \\
\hline EDGAR, 1956 & $\begin{array}{l}\text { Dovoinea proglottina } \\
\text { Choonotaenıa infundibulum } \\
\text { Rasllietino cesticillus }\end{array}$ & $\begin{array}{c}\text { U. S. A. } \\
- \\
-\end{array}$ & $\begin{array}{l}750 \\
750 \\
725\end{array}$ & $\begin{array}{r}0 \text { p. } 100 \\
100 \text { p. } 100 \\
94 \text { p. } 100\end{array}$ \\
\hline EDGAR ET TEER, 1957 & Raillietina cesticilius & - & 375 & 54 p. 100 \\
\hline $\begin{array}{c}\text { ENIGK ef DUWEL, } 1959 \\
-\end{array}$ & $\begin{array}{l}\text { Dovainea progiottina } \\
\text { Raillietino cesticillus }\end{array}$ & $\begin{array}{c}\text { Allemagne } \\
-\end{array}$ & $\begin{array}{l}500 \\
500\end{array}$ & $\begin{array}{l}0 \text { p. } 100 \\
0 \text { p. } 100\end{array}$ \\
\hline SAWADA, 1959 & $\begin{array}{l}\text { Roillietina cesticillus } \\
\text { Raillietina echinobothrida }\end{array}$ & $\stackrel{\text { Japon }}{-}$ & $\begin{array}{l}300 \\
300\end{array}$ & $\begin{array}{l}100 \text { p. } 100^{*} \\
100 \text { p. } 100^{*}\end{array}$ \\
\hline
\end{tabular}

* Si les parasites ne sont pas trop nombreux, sinon il subsisle quelques Cestodes.

40 Les formes immatures des espèces étudiées sont rarement atteintes et donnent des Cestodes adultes malgré l'intervention thérapeutique;

$5^{\circ}$ Le Dichlorophène est inefficace sur les Nématodes observés (Ascaridia styphlocerca, Subuluro brumpti et Acuaria spirolis);

6o ll n'y a pratiquement aucune différence dans l'action du Dichlorophène à 98 p. 100 et le même corps pur:

$7^{\circ}$ L'anthelminthique doit être administré en capsules de gélatine, car s'il est mélangé à la nourriture, son odeur aromatique éloigne trop de poulets ;

$8^{\circ}$ Les conséquences du traitement sur la santé des oiseaux sont faibles ou nulles; la mort ne survient qu'avec des doses de $4 \mathrm{~g} / \mathrm{kg}$, ce qui implique un coefficient chimiothérapique élevé de l'ardre de 5 à 13 suivant les doses utilisées.

\section{CONCLUSION}

Le Dichlorophène, quoique peu toxique, est un anthelminthique cestocide faiblement polyvalent, ce qui limite son emploi dans des régions comme l'Afrique tropicale (Tchad) où les formes adultes ef immatures de nombreux Cestodes (Choonotaenia infundibulum, Cotunio digonopora, Hymenolepis carioca, Raillietina tetragona, $R$. echinobothrido ef $R$. cesticillus) existent toute l'année avec divers Nématodes (Ascaridia styphlorcerca, Subulura brumpti et Acuoria spiralis) sur un très grand nombre d'oiseaux.

Ecole nationale vétérinare d'Alfort Laborotoire de parositologie

Laboratoire de recherches vétérinaires de Farcha-Fort-Lamy (Rép. du Tchad) Service de parasifologie

\section{SUMMARY}

\section{The action of dichlorophene on avian cestodes ad nematodes}

Dichlorophene, mildly toxic, is an anthelmintic, feebly polyvalent against the adult and immature forms of the common cestodes and nematodes of poultry in tropical Africa (Tchad). This limits its use in these parts since a great number of birds are heavily infested with many species which are detailed. 


\section{RESUMEN}

Accion del diclorofene sobre los cestodes y nematodes dël pollo

El diclorofeno, aunque poco tócixo, es un antihèlmíntico cestocida escasamente polivalente por lo que su empleo se ve limitado en regiones como el Africa tropical (Tchad) donde las formas aduitas e inmaduras de numerosos cestodes (Choanotaenia infundibulun, Cotunia digonopora. Himenolepsis carioca, Railletina tetragona, $R$. echinobotrida y $R$. cesticulus) existen todo el anos junto con diversos Nematodes (Ascaridia estiflacerca, Subulura brumpti y Acuaria espiralis) sobre un muy grande número de animales. 